\title{
Near-Relativistic Solar Electrons and Type III Radio Bursts
}

\author{
H. V. Cane \\ Laboratory for High Energy Astrophysics, NASA/GSFC, Greenbelt MD 20771
}

\begin{abstract}
Recently it has been found that the inferred injection times of $>25 \mathrm{keV}$ electrons are up to 30 minutes later than the start times of the associated type III radio bursts at the Sun. Thus it has been suggested that the electrons that produce type III bursts do not belong to the same population as those observed above $25 \mathrm{keV}$. This paper examines the characteristics and circumstances of 79 solar electron beam events measured on the ACE spacecraft. Particular attention is paid to the very low frequency emissions of the associated radio bursts and the ambient conditions at the arrival times of the electrons at the spacecraft. It is found that the inferred $>25 \mathrm{keV}$ electron injection delays are correlated with the times required for the associated radio bursts to drift to the lowest frequencies. This suggests that the electrons responsible for the radio emission and those observed above $25 \mathrm{keV}$ are part of a single population, and that the electrons both above and below $25 \mathrm{keV}$ are delayed in the interplanetary medium. Further evidence for a single population is the general correspondence between electron and local radio intensities and temporal profiles. It is found that the delays increase with the ambient solar wind density consistent with the propagation times of the electrons being determined by the characteristics of the interplanetary medium. However it is known that particle arrival times at $1 \mathrm{AU}$ are a linear function of inverse particle speed. Conventionally such a relationship is taken to indicate scatter-free propagation when inferred path lengths lie close to $1.2 \mathrm{AU}$, as they do for the electron events studied here. These conflicting interpretations require further investigation.
\end{abstract}

Subject headings: Sun: flares - Sun: particle emission - interplanetary medium

\footnotetext{
${ }^{1}$ Also School of Mathematics and Physics, University of Tasmania, Hobart, Tasmania, Australia
} 


\section{Introduction}

Much of what has been learned about electron acceleration at the Sun has been derived from observing the electromagnetic emissions that electrons generate so readily. Fast-drifting type III radio bursts, and the associated hard Xray bursts, are archetypal signatures of flare processes. The causative electrons are generally believed to be energized by direct electric field acceleration or stochastic acceleration by waves (e.g. Bastian et al. (1998)). Type III bursts are especially important because they trace electron paths that usually escape to the interplanetary medium. The emission is radiated at the local plasma frequency and its first harmonic. The downward frequency drift with time results from the propagation of electrons from the low corona into the interplanetary medium through a plasma that decreases in density. Ground-based radio observations above about $20 \mathrm{MHz}$ indicate the time when the electrons were within a few solar radii of the Sun's surface. Spacecraft experiments allow the bursts to be tracked through the interplanetary medium to the lowest frequencies ( 10 $\mathrm{kHz}$ ) which originate near $1 \mathrm{AU}$. The radio waves are a secondary product from Langmuir waves produced by the electron beam. In situ observations comparing low energy electrons, radio emissions and Langmuir waves (e.g. (Lin et al. 1981, 1986; Ergun et al. 1998; Buttighoffer 1998)) have demonstrated that the electrons responsible for type III bursts have energies in the range $2-20 \mathrm{keV}$. These observations have also shown that the Langmuir waves typically commence when the $\sim 10-20 \mathrm{keV}$ electrons are just arriving at $1 \mathrm{AU}$ and that the initial radio emission is nearly always at the fundamental. Because radio emission near the plasma frequency is strongly scattered and absorbed in the highly inhomogeneous interplanetary medium, radiation at the plasma frequency cannot propagate tangentially to the density gradient and the radiation has been found to be beamed along the local magnetic field direction (Reiner and Stone 1988). This means that when the electrons reach distances of the order of $1 \mathrm{AU}$ radio emission is seen just above the local plasma frequency only when the electron beam is very close to the spacecraft. If the radio burst does not extend to low frequencies $(<\sim 50 \mathrm{kHz}$ ) then the electron beam has missed the spacecraft and an in situ electron event is not observed (Cane and Erickson 2003). Otherwise the emission extends to the local plasma frequency unless the intensity of the electrons is too low relative to the background population to produce emission. Near $1 \mathrm{AU}$ the local plasma frequency varies from about 7 to $50 \mathrm{kHz}$ and is typically near $25 \mathrm{kHz}$.

Recently several authors have found delays between electromagnetic emissions from flares and the inferred injection times of $>25 \mathrm{keV}$ electrons at the Sun. The calculated injection times are based on the arrival times of the electrons at $1 \mathrm{AU}$ and making the assumption that there is no scattering whatsoever in the interplanetary medium. The primary flare signature used for a solar fiducial mark is the type III radio burst - known to be caused by electrons. To account for the delay between the onset of the Type III burst and the $>25$ 
$\mathrm{keV}$ electron injection, Krucker et al. (1999) proposed that there are two separate electron populations and that the one that produces the radio burst did not intercept the spacecraft. A similar suggestion has been posited by Klassen et al. (2002). Such a suggestion implies that the population that was detected did not produce a radio burst at the Sun.

This scenario was also implied by the work of Haggerty and Roelof (2002) who focused on events with very anisotropic electron distributions beamed along the magnetic field direction. For such events Haggerty and Roelof (2002) assumed that interplanetary propagation effects would be negligible. The present study considers the properties of these same electron 'beam' events. A detailed analysis is made of the solar associations of the events, the interplanetary conditions at their time of observation, and the properties of the associated type III radio bursts. In particular it is demonstrated that the times required for the radio bursts to drift from $>25 \mathrm{MHz}$ to, or close to, the local plasma frequency are correlated with the deduced $>25 \mathrm{keV}$ electron delays. It is also shown that the delays are related to the density of the interplanetary medium and that, furthermore, the vast majority of the beamed events occur in plasma with unusually low densities. The significance of these results

is that the radio emission, which traces the propagation of the electrons responsible for the emission, directly shows that electron travel time from the Sun to the Earth is variable and is longer than would be expected based upon direct propagation along a smooth $1.2 \mathrm{AU}$ field line. The fact that the more energetic electrons suffer delays which are correlated with those at lower energies, and that these are associated with interplanetary conditions implies that both type III-producing and the more energetic electrons are affected by propagation conditions in the interplanetary medium. The observations strongly support the suggestion that inferred delays between Type III bursts and $>25 \mathrm{keV}$ electron injection at the Sun occur in the interplanetary medium.

\section{Data Analysis}

Currently there are two spacecraft near $1 \mathrm{AU}$ with experiments observing electrons at energies below $0.3 \mathrm{MeV}$. The Wind 3-D Plasma and Energetic Particle experiment (Lin et al. 1995) makes observations from solar wind energies to several hundred $\mathrm{keV}$. The two detector systems of interest to the present study cover the energy ranges $0.4-26 \mathrm{keV}$ and 27-311 keV. The signal-to-noise ratio for the lower energies is relatively low and thus fewer electron events are observed at these energies. The EPAM experiment on ACE (Gold et al. 1998) measures electrons in four energy channels in the range $38-315 \mathrm{keV}$. Thus this experiment does not measure electrons at the $2-20 \mathrm{keV}$ energies responsible for type III radio bursts. The SWEPAM experiment on ACE (McComas et al. 1998) measures electrons from 
$1.6 \mathrm{eV}$ (thermal energies) to $1.35 \mathrm{keV}$.

Researchers from both the Wind and EPAM experiment teams (Krucker et al. 1999; Haggerty and Roelof 2002) have compared electron events at $1 \mathrm{AU}$ with radio bursts measured near the Sun. In particular they have examined radio bursts reported from groundbased measurements and from the WAVES experiment (Bougeret et al. 1995) on Wind. The WAVES experiment has three receivers, covering different frequencies ranges, namely RAD2 ( 2-14 MHz), RAD1 (20-1040 kHz) and, TNR (4-245 kHz). The full frequency range covers emissions generated from about 2 solar radii all the way to $1 \mathrm{AU}$. Both research teams find an excellent correlation between the occurrence of electron events detected near Earth and type III bursts seen by the WAVES experiment. When comparing electron injections at the Sun and radio bursts Krucker et al. (1999) and Haggerty and Roelof (2002) primarily considered observations from the RAD2 receiver. Haggerty and Roelof (2002) first determined the arrival time of the electrons at $1 \mathrm{AU}$, then calculated when they left the Sun by subtracting an interval they believed would be the transit time of the electrons under scatter-free propagation along a Parker spiral field line. They then compared this time with the time of peak $14 \mathrm{MHz}$ emission (i.e. the upper frequency of the RAD2 receiver). Such a calculation leads to the result that the $>25 \mathrm{keV}$ electrons left the Sun 1-30 minutes after the lower energy electrons which generated the $14 \mathrm{MHz}$ radio emission. Haggerty and Roelof (2002) therefore concluded that the $>25 \mathrm{keV}$ electrons were accelerated later and by a different process than the type III-producing electrons.

In this paper a different approach is taken; the transit times of the $>25 \mathrm{keV}$ electrons to $1 \mathrm{AU}$ are compared with the transit times of the radio generating electrons. It will be shown that these times are correlated. The radio electron transit times are calculated by following the leading edge of the radio burst as seen in the WAVES TNR data. The TNR receiver was specifically designed to study the plasma thermal noise generated in the antenna as a result of local plasma oscillations (Meyer-Vernet and Peche 1989). This noise is responsible for a black band (called the "plasma line") at the bottom of dynamic spectra (see Figure 1). Its peak frequency gives the local plasma frequency and hence local plasma density. Since radio emission cannot propagate below the local plasma frequency there is an intrinsic cut-off in the radiation at the plasma line. The intercept of the leading edge of a type III radio burst and the plasma line indicates when the responsible electrons have arrived at the spacecraft. A statistical analysis has been made of the times taken by the bursts to drift from high frequencies to very low frequencies (close to the plasma line) for most $(61 / 79)$ of the events in the Haggerty and Roelof (2002) list. This analysis was not possible for some bursts caused by low intensity beams in which the radiation cut-off well above the plasma line or bursts in which the low frequency part was obscured by other radio emissions. This technique has previously been applied by Cane and Erickson (2003) to intense bursts 
associated with major solar particle events seen both at $1 \mathrm{AU}$ and Ulysses and the method is illustrated in more detail in that paper. In the Cane and Erickson (2003) study many of the particle events considered were not as well magnetically connected to the spacecraft as the electron beam events. Thus some of the associated radio bursts took much longer to drift to the plasma frequency than the bursts associated with electron beam events.

Figure 1 shows two examples of the relative timing between radio emissions and electron increases. The upper panel of each pair shows 5-minute averaged electron data from ACE in two energy ranges (38-53 and 175-315 keV) and the bottom panels show the TNR radio data. The type III bursts are shown in two ways. The grey shading shows the receiver voltages (1 minute averages) as a function of frequency and time. Superimposed are a few profiles at individual frequencies. (The profiles are only approximately at the frequencies indicated on the frequency axis in order that the relative levels can be discerned.) The vertical lines on the radio plots delineate hours of UT. The plots start exactly 30 minutes before the reported start of the associated type III seen from the ground; this time is listed at the top of the electron panels and does not take account of the time for the radio emission to propagate to 1 AU. The plasma line is the faint grey, almost horizontal, line that lies at frequencies just below the radio bursts. Visual estimates of the times of the interceptions of the leading edges of the radio bursts and the plasma line are indicated by heavy black lines below the graphs. It is clear that the radio bursts in these examples have very different drift rates. The May 2000 radio event drifted to the lowest frequencies in about 30 minutes - or 38 minutes taking into account that the highest frequency radio emission takes 8 minutes to travel from the sun to 1 AU. In contrast the September 1998 radio burst took about 90 minutes. If it is assumed that the responsible electrons were always at the same energy and traveled along a 1.2 AU path length with no scattering, then these drift times would imply that electrons of about $20 \mathrm{keV}$ and $4 \mathrm{keV}$ produced the radio emission in these two events. In the case of the May 12000 event there is evidence of Langmuir waves seen as rapid intensifications on the plasma line. The first time they are seen is slightly before $1100 \mathrm{UT}$. At this time the Wind experiment shows that $\sim 20 \mathrm{keV}$ electrons were starting to arrive (data not shown). The ACE electron intensity profiles shown in Figure 1 are also clearly different, with the May 2000 event rising rapidly whereas the September 1998 event increased more gradually and started later relative to the solar event.

The time interval between the start of the type III radio burst at the Sun and the first electron increase above background in the ACE EPAM data was determined for all of the events in the study. For the two events of Figure 1, these intervals were 17 and 33 minutes. The radio burst times at the Sun were based on ground-based observations as reported in Solar Geophysical Data. Apart from 7 events for which there were no radio observations, all but two of the electron beam events (i.e. $70 / 72(97 \%)$ ) could be associated with type III 
activity observed above $25 \mathrm{MHz}$. The electron arrival times, based on high time resolution data, are listed in Haggerty and Roelof (2002). They used different electron channels for different events depending on their intensities. For the events of Figure 1 Haggerty and Roelof (2002) estimated that the relevant electrons would have taken about 14 minutes and 16 minutes to travel from the Sun to $1 \mathrm{AU}$. Therefore these events are deduced to have had 'delayed' injections relative to the type III bursts of 3 and 17 minutes. Figure 2 shows the radio drift times as a function of 'injection delays', calculated this way, for 61 of the 79 events. Errors of \pm 3 minutes have been allocated to the electron times based on differences between injection times determined by the Wind and ACE research teams. (The Wind team determine injection times differently as will be discussed later.) The radio drift time is defined as the interval between the start time of the type III burst at the Sun and when its leading edge reached the plasma line. The technique was to follow the leading edge of the burst over the lowest $\sim 20 \%$ of the frequency extent from the top of the TNR range $(245 \mathrm{kHz})$ down to the plasma frequency. The radio burst onsets at low frequencies are not sufficently well defined (for various reasons including intrumental problems) to warrant a more elaborate analysis. Generous errors were allocated to the intercept time. Even in the cases when there was a small gap between the plasma line and when the radio emission ceased extrapolation to the plasma line was possible. If emission ceased too far above the plasma line then the event was omitted from the analysis. In Figure 1 of the Cane and Erickson (2003) paper several examples are shown with dashed lines indicating the slopes of the leading edges of the bursts. Although the technique is crude it is obvious from Figure 1 that there are significant differences in the drift rates of bursts. Figure 2 shows that radio drift times and electron injection delays are clearly correlated, with a correlation coefficient of 0.81 . Such a correlation would be extremely improbable if type-III producing and energetic electrons were two independent populations - there is no obvious reason why a long radio drift time, resulting from electron propagation in the interplanetary medium between the Sun and $1 \mathrm{AU}$, should also be associated with a long delay between the type III burst and energetic electron injection at the Sun. However, it is to be expected if there is only one electron population involved, and both type-III producing and energetic electrons within this population are delayed by propagation conditions in the interplanetary medium, increasing the radio drift time and inferred "injection delay" in concert.

As noted in reference to Figure 1 there is some correspondence between the electron and radio intensity-time profiles. Furthermore, there is also some general correspondence in their intensities; the larger electron increase has a more intense radio burst. Figure 3 illustrates this more clearly for two events (see figure caption for details of radio spikes). These events were chosen to occur in similar solar wind densities (proton density of $3 \mathrm{~cm}^{-3}$ ) so that the same frequency $(19.7 \mathrm{kHz})$ corresponds to just above the local plasma frequency 
$(15.6 \mathrm{kHz}$ ) for both events. Thus the radio emission produced near $1 \mathrm{AU}$ is compared with the electron beam population at $38-53 \mathrm{keV}$. Similar correspondences are found in comparing other bursts although sometimes the correspondence is not as good. This is likely a result of the fact that the radio emission is caused by electrons with energies below the $38-53 \mathrm{keV}$ EPAM channel; if the spectrum is relatively steep/flat the radio emission could be relatively more intense/weak when compared with the $38-53 \mathrm{keV}$ electron intensity.

Since the results in Figure 2 suggest that the electron delays occur in the interplanetary medium, a study was made of the solar wind conditions at the time when the particles arrived at the spacecraft. It was found that the solar wind density provided some organization of the data as shown in Figure 4. The inferred injection delays at the Sun for the 41 larger (38-53 $\mathrm{keV}$ electron intensity $\geq 10^{4}$ particles $/\left(\mathrm{cm}^{2}\right.$-sec-ster-keV)) electron events (filled circles) are correlated with the local plasma densities with a coefficient of 0.66 . The triangles represent events with lower electron intensities. If the injection delays occurred at the Sun it is unlikely that they would be related to the solar wind density at $1 \mathrm{AU}$.

The very largest events occurred in very low density solar wind and had the fastest rise times. What is very significant is that the mean density at the time of the electron beam events with data $(75 / 79)$ was $4.3 \mathrm{~cm}^{-3}$ which is below the mean density of $\sim 7 \mathrm{~cm}^{-3}$ of the entire solar wind as determined from ACE Level 2 data. Since most solar particle events occur in slow solar wind it is probably more relevant to compare with its density of $\sim 9 \mathrm{~cm}^{-3}$. In this case the densities at the times of beamed electron events are even more unusual. A preliminary study was made to determine if non-beamed electron events occur preferentially in solar wind of higher densities. This was found to be the case with $\sim 83 \%$ of non-beam events occurring in solar wind of densities $>5 \mathrm{~cm}^{-3}$ compared with only $28 \%$ of beamed events.

\section{Discussion}

A number of authors who have studied solar energetic electron events, including Haggerty and Roelof (2002) and Krucker et al. (1999), have suggested that the population of energetic electrons observed at Earth is different from that which produces the type III radio bursts observed at approximately the same time. In the present paper it is shown that the associated radio bursts drift to frequencies that are produced at or very near the spacecraft with rates that differ from event to event and that the drift rates are correlated with the deduced electron delays at the Sun (see Figure 2). The close correspondence between the electron and radio emission intensities evident in Figures 1 and 3 also provides strong support for the suggestion that there is only a single electron population. Furthermore, an investiga- 
tion of the interplanetary conditions at the onset times of the beamed electron events found that they all occurred in relatively low density solar wind and that the electron propagation times tended to increase with increasing density. A related result is that of Buttighoffer (1998) who found that low frequency type III bursts, Langmuir waves and electron events were observed at Ulysses only when the spacecraft was inside "propagation channels". These channels were characterized by very quiet plasma and magnetic field parameters. Buttighoffer (1998) suggested that the very low level of magnetic field fluctuations was the important feature for particle propagation. An initial investigation of high time resolution magnetic field intensities and directions at the times of the ACE electron beam events did not confirm this result but an extensive investigation is beyond the scope of the present study.

As mentioned above, Krucker et al. (1999) did not just consider electron arrival times at a single energy, as did Haggerty and Roelof (2002), but considered the full range of energies from their experiment. For events only observed above $25 \mathrm{keV}$ they determined a path length assuming no scattering and then shifted the time profiles in each energy range by the appropriate amount to take into account the time of flight to near Earth. The profiles were found to lie on top of each other and the release time at the Sun was taken to be the time when the normalized, background-subtracted profiles rose above zero. For 12 events observed below $25 \mathrm{keV}$ Krucker et al. (1999) plotted onset times for each energy range as a function of inverse electron speed. The expectation is that the slope of such a plot gives the path length whereas the intercept gives the injection time. Krucker et al. (1999) presumably obtained path lengths near 1.2 AU but specific path lengths are not given in the paper. When onset times vary in a linear fashion with inverse electron speed and imply path lengths compatible with a smooth Parker spiral field this seems to imply scatter-free propagation. Also the highly beamed electron events selected by Haggerty and Roelof (2002) seem to imply scatter-free propagation. However the above analysis of the associated radio emission shows that this unlikely to be correct. The analysis implies that there is only one electron population and, from a comparison of the radio emission and electron event characteristics, that population takes longer than expected to travel to Earth. It is important to note that in 10 of 12 cases with low energy observations studied by Krucker et al. (1999) the onset times for electrons over the full range, $\sim 300 \mathrm{keV}$ down to below 1 $\mathrm{keV}$, fell on the same straight line with no indication for two populations. Also there is no indication in the electron intensity-time profiles for two populations.

To resolve the discrepancy between the two types of analysis will require investigations using transport models and detailed anisotropy information. An initial study of this kind was undertaken by Kallenrode and Wibberenz (1990). They found linear relationships between particle onsets and inverse particle speed and inferred delayed injections. The injection duration at the Sun was one parameter causing delays. However, based on durations of type 
III bursts at the Sun, the injection times considered by Kallenrode and Wibberenz (1990) may not be appropriate for the majority of the electron beam events considered here.

\section{Conclusions}

Data are presented that support the proposal that there exists a single energetic electron population that both produces type III radiation as it is released from the Sun and while it propagates to the vicinity of the Earth, where it is observed directly. Interaction effects in the interplanetary medium must cause the "anomalous" delays between the inferred release of the electrons from the Sun and the type III emission generated near the Sun.

I am grateful to W. C. Erickson and G. Wibberenz for continuing my education about radio emission and particle propagation, respectively, and thank I. G. Richardson and S. Krucker for a critical reading of the manuscript. I appreciate the use of the data made available via the NSSDC CDAWeb. This work was partially funded by a NASA contract with USRA. 


\section{REFERENCES}

Bastian, T. S. et al. 1998, Ann. Rev. Astron. Astrophys., 36, 131

Bougeret, J.-L., et al. 1995, Space Sci. Rev., 71, 231

Buttighofer, A. 1998, Astron. Astrophys., 335, 295

Cane, H.V. et al. 2002, J. Geophys. Res., 107(A10), 1315, doi:10.1029/2001JA000320

Cane, H. V. and W. C. Erickson 2003 J. Geophys. Res., 108(A5), 1203, doi:10.1029/2002JA009488

Dulk, G. A., et al. 1998, J. Geophys. Res., 103, 17,223

Ergun, R. E., et al. 1998, ApJ, 503, 435

Gold, R. E. et al. 1998, Space Sci. Rev., 86, 541

Haggerty, D. K. and Roelof, E. 2002, ApJ, 579, 841

Kallenrode, M.-B, and Wibberenz, G. 1990 Proc. 21st Int. Cosmic Ray Conf., 5, 229

Klassen, A. et al. 2002, Astron. Astrophys., 385, 1078

Krucker, S., et al. 1999, ApJ, 519, 864

Lin, R. P., et al. 1981, ApJ, 251, 364

Lin, R. P., et al. 1986, ApJ, 308, 954

Lin, R. P., et al. 1995, Space Sci. Rev., 71, 125

McComas, D. J., et al. 1998, Space Sci. Rev., 86, 563

Meyer-Vernet, N. , and Peche, C. 1989 J. Geophys. Res., 94, 2405

Reiner, M. J. and Stone, R. G. 1988, Astron. Astrophys., 206, 316 

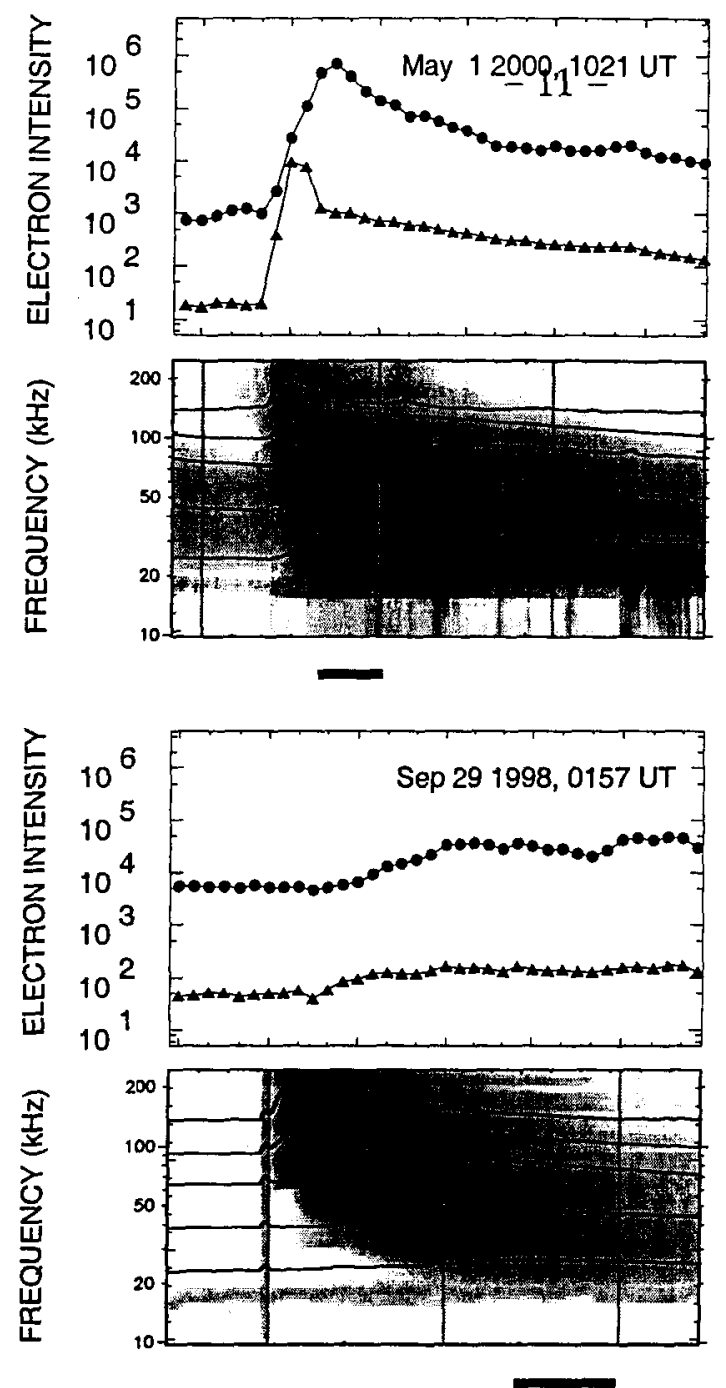

Fig. 1.- Comparison of electron and radio data for two events with very different deduced electron and radio delays. The 5-minute averaged electron data are from ACE and intensities in two energy ranges (38-53 and 175-315 keV) are shown as dots and triangles respectively. The units are particles $/\left(\mathrm{cm}^{2}\right.$-sec-ster-MeV). The start time of each plot is 30 minutes before the start of the radio burst at the Sun, which is given at the top of each particle panel. (Note that this is the time as observed at $1 \mathrm{AU}$ and so 8 minutes need to be subtracted to calculate the emission time at the Sun.) The grey vertical lines are at integral hours of UT. The radio data shows intensities from the Wind WAVES TNR receiver in the frequency range $10-200 \mathrm{kHz}$. The lines superimposed on the grey shaded spectrum are individual profiles for a few frequencies. The grey intensification across the bottom of the radio plots is the plasma thermal noise which is seen at a frequency which corresponds to the local plasma frequency. The times (with an uncertainty) that the leading edges of the bursts intercept the plasma line are indicated by the horizontal bars. 


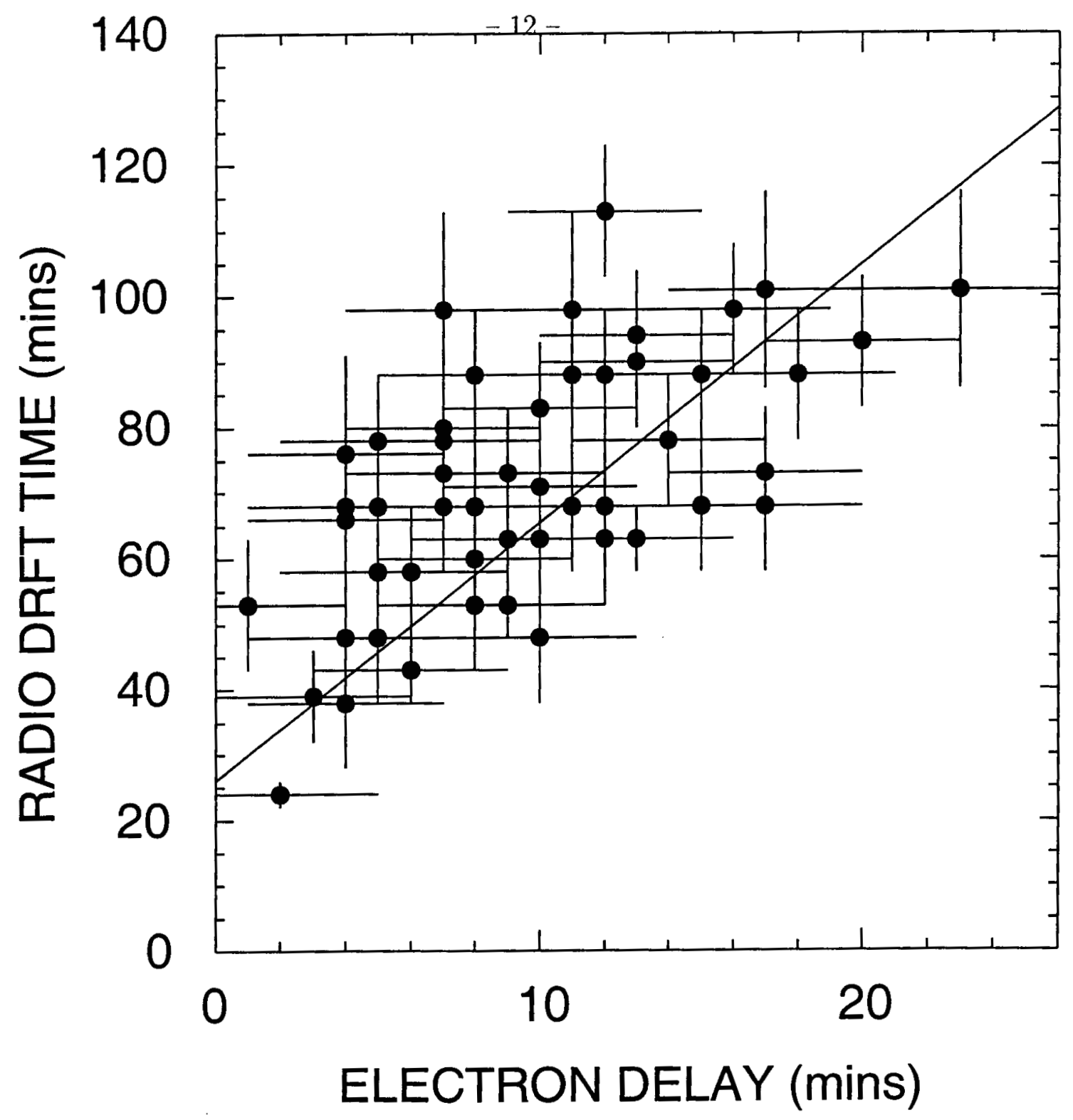

Fig. 2. - Radio drift times as a function of energetic electron delays. The radio drift times correspond to the times taken for the leading edges of the bursts to reach the plasma line following the start of emission above $25 \mathrm{MHz}$ as seen from Earth. Electron delays are the difference between the transit times of the electrons (relative to when the high frequency radio emission left the Sun) and the expected transit times if the electrons travel directly along a 1.2 AU field line without scattering. The plot shows that the travel times of the high energy electrons are correlated with the travel times of the lower energy electrons which generate the radio emission, suggesting that they belong to the same population. 

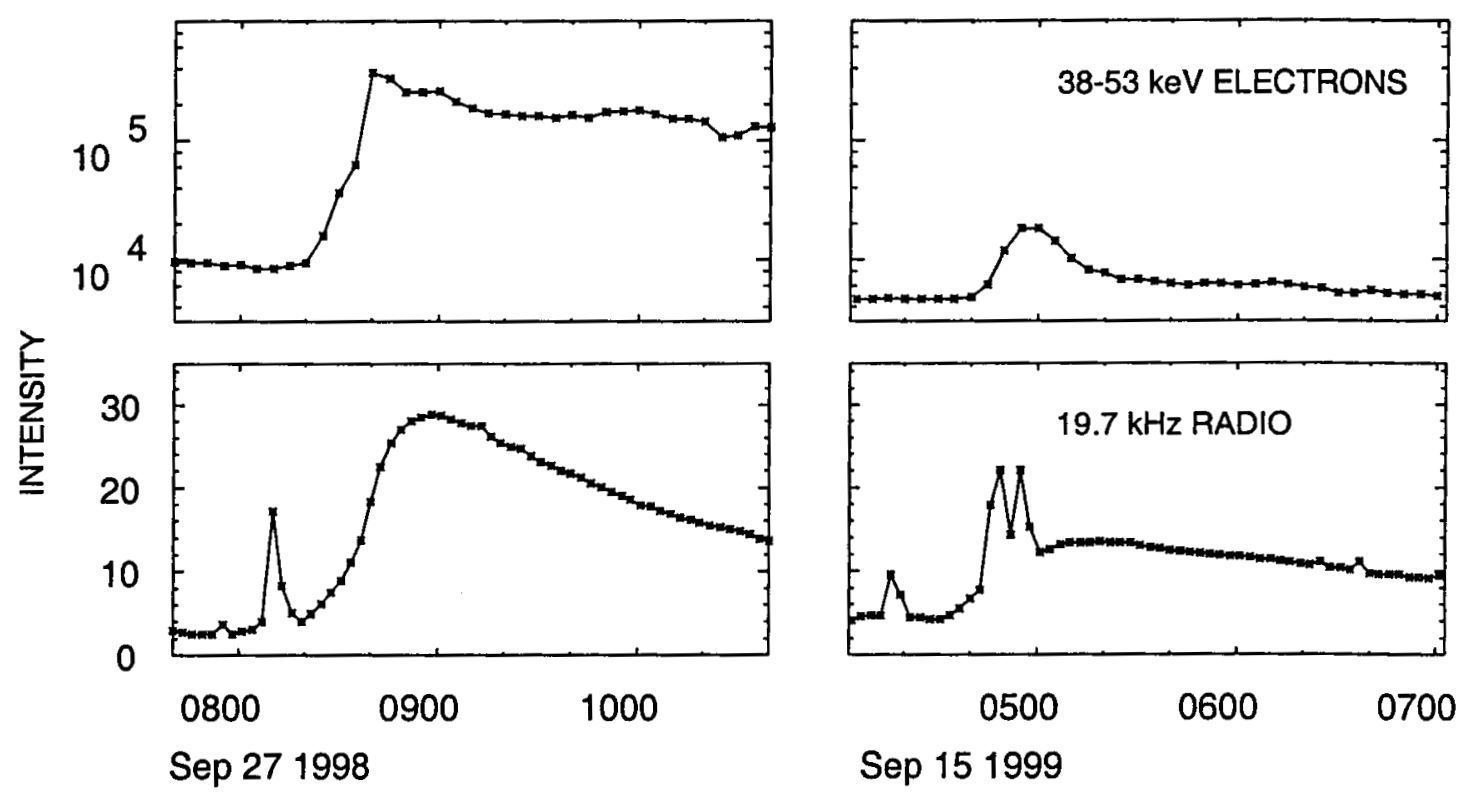

Fig. 3.- A comparison of $38-53 \mathrm{keV}$ electron intensities with the radio intensities produced relatively near the spacecraft for two events. The units are electrons $/\left(\mathrm{cm}^{2}\right.$-ster-sec-MeV) and $\mathrm{dB}$ above background. The large spike before the onset of the radio emission for the Sept. 1998 event is instrumental overloading caused by the strong higher frequency emission at this time. The spikes on the other event are possibly Langmuir waves. 


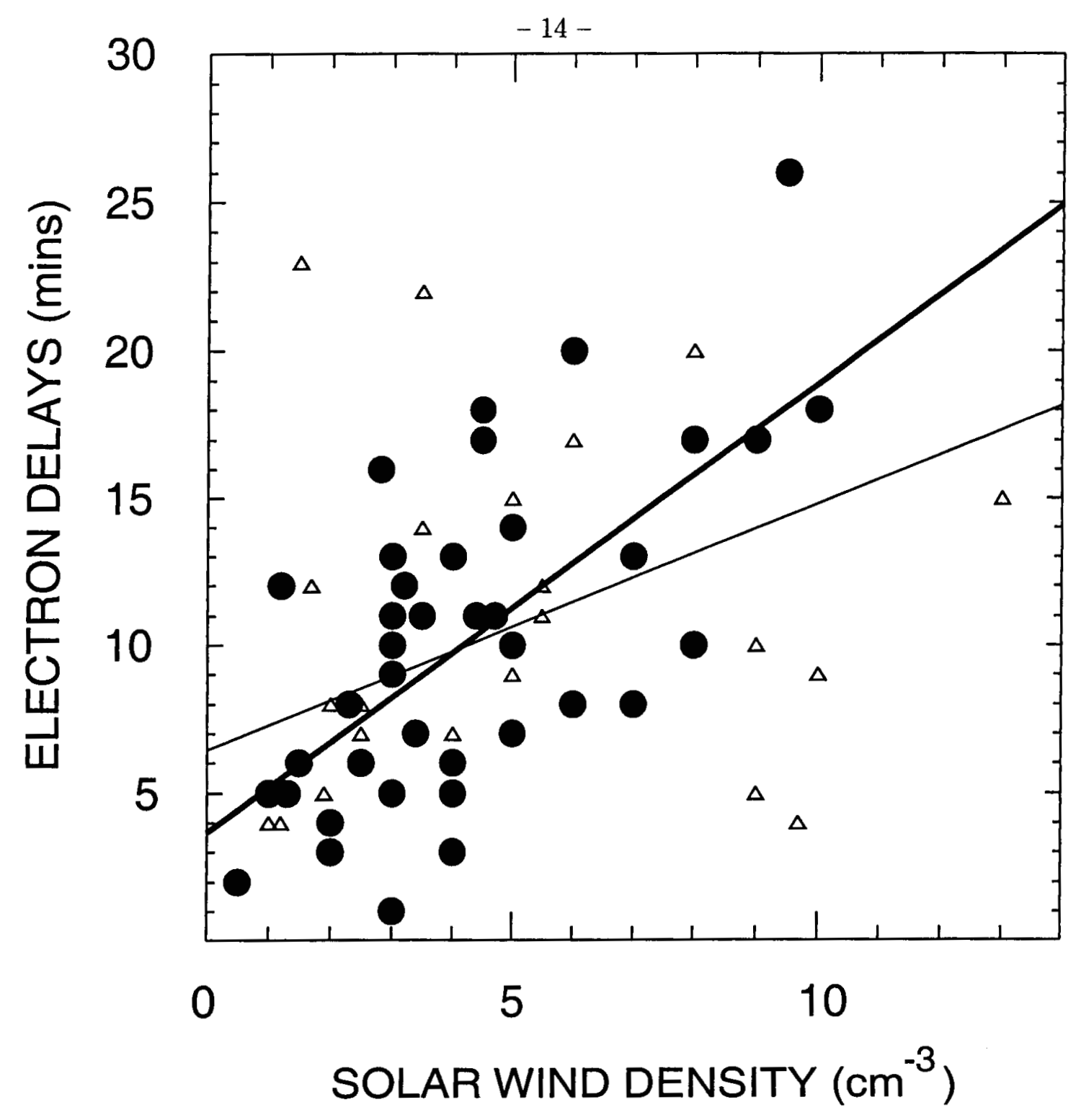

Fig. 4.- Correlation between the inferred electron delays and the local solar wind proton densities at the start of the electron events. The filled circles are for the more intense events and the thicker straight line is fitted to these 41 events. The correlation coefficient is 0.66 . The thinner line is a fit to all 70 events with a correlation coefficient of 0.41 . Triangles are used for events with intensities $<10^{4}$ particles $/\left(\mathrm{cm}^{2}\right.$-sec-ster-keV). Note that the mean density of the solar wind is about $7 \mathrm{~cm}^{-3}$, above the densities at the times of most of the events. 